\title{
A numerical study of fixed frequency reflectometry measurements of plasma filaments with radial and poloidal velocity components ${ }^{\text {a) }}$
}

\author{
J. Vicente, ${ }^{1, b)}$ F. da Silva, ${ }^{1}$ S. Heuraux ${ }^{2}$, M.E. Manso ${ }^{1}$, G.D. Conway ${ }^{3}$, C. Silva ${ }^{1}$ and \\ the ASDEX Upgrade team ${ }^{3}$
}

\author{
${ }^{1}$ Instituto de Plasmas e Fusão Nuclear, Instituto Superior Técnico, Universidade de Lisboa, 1049-001 Lisboa, \\ Portugal \\ ${ }^{2}$ Institut Jean Lamour, UMR 7198 CNRS-University Lorraine, Vandoeuvre, France \\ ${ }^{3}$ Max-Planck-Institut für Plasmaphysik, 85748 Garching, Germany
}

\begin{abstract}
(Presented XXXXX; received XXXXX; accepted XXXXX; published online XXXXX)
A 2D finite-differences time-domain full-wave code is used to simulate the measurements of plasma filaments with fixed frequency O-mode reflectometry. The plasma is modeled by a linear slab plasma plus a Gaussian perturbation propagating in a direction that can vary from poloidal to radial. The plasma background density gradient is chosen in agreement with the steep edge transport barrier of the ASDEX Upgrade H-modes. Illustrative results are presented and different types of reflectometry responses are observed depending on filament sizes and propagation directions. The reflectometry signatures obtained here with numerical simulations support previous experimental findings on filament measurements.
\end{abstract}

\section{INTRODUCTION}

Plasma filaments are coherent structures of high plasma density and have been observed in the edge and Scrape-Off Layer (SOL) plasmas of various magnetic fusion devices ${ }^{1}$. These structures are extended in the direction of the magnetic field lines and form a density blob that in medium sized tokamaks typically exhibit poloidal sizes of some centimetres and somewhat smaller radial sizes. Filaments originate in a region somewhere from the top of the pedestal to the vicinity of the Last Closed Flux Surface (LCFS) depending on the confinement mode and ultimately the trigger mechanism. A fraction of the plasma filaments travel with a radial velocity component across the Scrape-Off Layer (SOL) and can deposit their remaining energy and particle content in the plasma facing components. Filaments with densities of up to $80 \%$ of the pedestal top density have been measured in the far- $\mathrm{SOL}^{2}$. Microwave reflectometry is a radar-like technique that has been extensively used to measure electron density profiles and fluctuations in fusion devices ${ }^{3}$. At the ASDEX Upgrade tokamak (AUG) a Fixed Frequency Reflectometry (FFR) system with ordinary wave polarization (O-mode) has been recently used to measure filamentary activity in the vicinity of the LCFS in ELMy $\mathrm{H}$-mode plasmas ${ }^{4}$. Since wave propagation in a perturbed fusion plasma can be quite complex, and to better assess the capabilities of the experimental technique, we perform a numerical study of the reflectometry response to elementary filament activity. We employ a 2D finite-difference time-domain (FDTD) full-wave code and a synthetic reflectometer to mimic the basic experimental conditions met at AUG. Filaments are modeled as Gaussian density perturbations with the characteristic dimensions found in medium sized tokamaks and we study their propagation with different velocity components ranging from purely poloidal to purely radial.

a) Contributed paper published as part of the Proceedings of the 20th Topical Conference on High-Temperature Plasma Diagnostics, Atlanta, Georgia, June, 2014.

b) Author to whom correspondence should be addressed:

jvicente@ipfn.ist.utl.p

\section{THE CODE AND SIMULATION SET-UP}

The code used in this work (REFMUL) solves the Maxwell equations for an O-mode wave that are coupled to the plasma electron density through the current density equation (see F. da Silva ${ }^{5}$ ). The plasma is thus modeled solely by the electron density $n_{e}(r, t)$. The simulation box comprises a rectangular grid with dimensions $\mathrm{L}_{\mathrm{x}}=1265$ and $\mathrm{L}_{\mathrm{y}}=2000$ (in grid points) corresponding to $\approx 47 \times 75 \mathrm{~cm}$, where $\mathrm{L}_{\mathrm{x}}$ and $\mathrm{L}_{\mathrm{y}}$ are the radial and poloidal directions in the usual tokamak coordinate system. The plasma itself measures only $\approx 10 \times 75 \mathrm{~cm}$ since part of the simulation box is for the antenna setup and a vacuum distance to the plasma. The real spatial dimensions of the tokamak edge region can be simulated with the code but full temporal simulations are not feasible due to Courant-Friedrichs-Lewy ${ }^{6}$ conditions that impose a small time step discretization. As a consequence, the time duration of any simulated phenomena has to be scaled down, and any velocities involved scaled up. In order to avoid relativistic effects we insure that the resulting scaled-up velocities do not exceed values $\approx 10 \%$ of $c$, the speed of light ${ }^{7}$. A unidirectional transparent source is used for injection of the signal that allows separating the emitted probing wave from any returning waves ${ }^{5}$. For directivity a monostatic 2-D H-plane horn antenna is used with a half-power beam width of $\approx 6.5 \mathrm{~cm}$ at the plasma entry. The selected probing wave has frequency $f_{o}=49.4 \mathrm{GHz}$ and wavelength in vacuum $\lambda_{0} \approx 0.6 \mathrm{~cm}$, corresponding to a critical density of $3.01 \times 10^{19} \mathrm{~m}^{-3}$. This corresponds to the minimum probing frequency of the V-band channel of the FFR system at $\mathrm{AUG}^{8}$. The AUG system is equipped with an I-Q (inphase/quadrature) detection scheme that allows to extract both the phase $\varphi(t)$ and amplitude $A(t)$ of the reflected signals. We can simulate the I-Q detection obtaining the signals $\mathrm{I}(t)=A(t) \cos [\varphi(t)]$ and $\mathrm{Q}(t)=A(t) \sin [\varphi(t)]$ which can then be combined to give $A(t)=\left[\mathrm{I}^{2}(t)+\mathrm{Q}^{2}(t)\right]^{1 / 2}$ and $\varphi(t)=\operatorname{atan}[\mathrm{Q}(t) / \mathrm{I}(t)]$.

\section{THE PLASMA MODEL AND DYNAMICS}


The plasma is modeled by a slab unperturbed background plasma with radial linear electron density profile plus a superimposed perturbation (the plasma filament). We have not considered broadband plasma turbulence. The linear background profile was chosen with a density gradient length $L \approx 2 \lambda_{0}$ in agreement with steep gradients found in the vicinity of the LCFS in inter-ELM periods of AUG plasmas. Typically, this region is probed by the FFR at AUG in those periods. For the filament model we use Gaussian density profiles in both radial and poloidal directions which are a good approximation to experimental observations. Additionally, a flat-top extension can also be used for reasons explained in the following paragraphs. Filaments (in 2D) propagate in the plasma with a velocity $V_{f i l}=\left(V_{r}^{2}+V_{\theta}^{2}\right)^{1 / 2}$ that can range from purely radial to purely poloidal. The pure radial propagation of Gaussian density perturbations is parametrized with built-in Gaussian functions of REFMUL. The pure poloidal propagation of filaments is simulated using a density matrix (with the total 2D plasma density structure envisaged) that is fed into the code which in turn slides it across the simulation box in the poloidal direction as the simulation progresses. In the cases where a single velocity component is considered the velocity magnitude is simply given by $V_{c o}=L_{c o} / \mathrm{N}_{\text {mov }}$ where $\mathrm{N}_{\text {mov }}$ is the number of iterations during which the propagation occurs and $L_{c o}$ is the total displacement in the direction of propagation. In order to simulate a perturbation propagating with both radial and poloidal velocity components we use a density matrix where the filament is modeled with an elongation in a certain direction tilted by an angle $\alpha$ from the poloidal axis. Then, as in the poloidal propagation case, the matrix is fed to the code and forced to move in the poloidal direction. In the reflectometry frame the result is a perturbation moving with a poloidal velocity component (of magnitude set as before) and a radial velocity component given by $V_{r}=V_{\theta} / \tan (\alpha)$. In this work the number of iterations comprising spatial displacement is $\mathrm{N}_{\mathrm{mov}}=200000$. As the poloidal length of the density matrix is kept constant $\left(L_{v, M}=4000\right.$ grid points $)$ the poloidal velocity component is also kept constant whith $V_{\theta}=$ $12 \times 10^{6} \mathrm{~m} . \mathrm{s}^{-1}$, i.e. $V_{\theta} / \mathrm{c} \approx 4 \%$. It was shown in previous studies 9 that a rescaling is possible while keeping invariant the results expressed as a function of the coordinate following the motion.

\section{RESULTS}

\section{A. Radial propagation}

The radial propagation is considered only in the outward direction i.e. down the density profile. For simplicity the ratio between the filament central density and the background density is kept constant. In fig. 1 we show density profiles (at the line of sight of the antenna) for several filaments of equal size but with different amplitude at their starting radial position. The position of the cut-off density $n_{e c}=3.01 \times 10^{19} \mathrm{~m}^{-3}$ (for $f_{o}=49.4 \mathrm{GHz}$ ) is used as a reference in the radial axis. The starting position of the filaments and the cut-off position correspond, respectively, to the pedestal top and the LCFS in the experiments. In fig. 2 we present the reflectometry signals (amplitude, phase and its time derivative) when probing the filaments depicted in fig. 1 (radial width $w_{r}=0.3 \mathrm{~cm}$ ) propagating radially. Note that $w_{r}$ and $w_{\theta}$ are used here as the standard deviation values of the Gaussian probability density function in each corresponding direction. The poloidal width in this case extends to the whole simulation box $(75 \mathrm{~cm})$. The results show the great reflectometry sensitivity to filaments with relatively small radial width provided a large poloidal size is used. The obtained phase signals behave as predicted by $1 \mathrm{D}$ geometric optics since they replicate the radial displacements of the cut-off layer: for higher density amplitudes the filament is able to push the cut-off layer further out before the filament density drops below the cut-off density as it propagates outwards. The phase then goes back to the unperturbed value and this also results in peaks in the time derivative of the phase that are sharper with increasing filament amplitudes. Simultaneously we observe a drop in amplitude that corresponds to complex electromagnetic flux conservation processes and wave interferences.

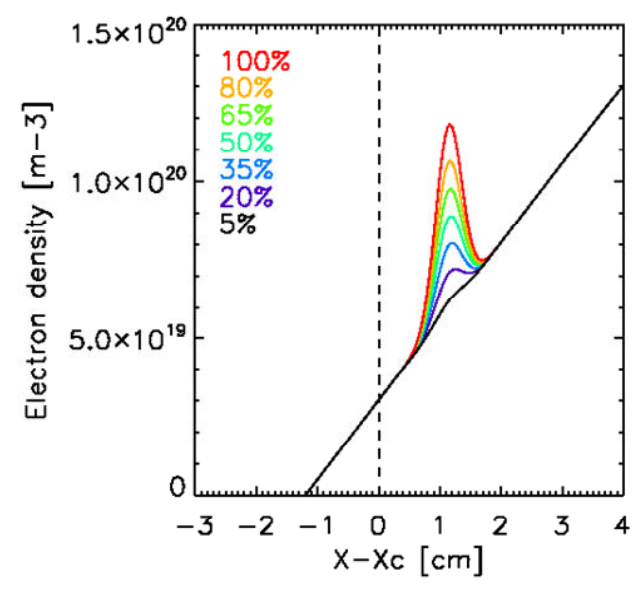

FIG.1. Initial density profiles for a set of Gaussian perturbations of varying amplitude used in radial propagation simulations. The cut-off layer corresponding to $f_{o}=49.4 \mathrm{GHz}$ is used as reference.

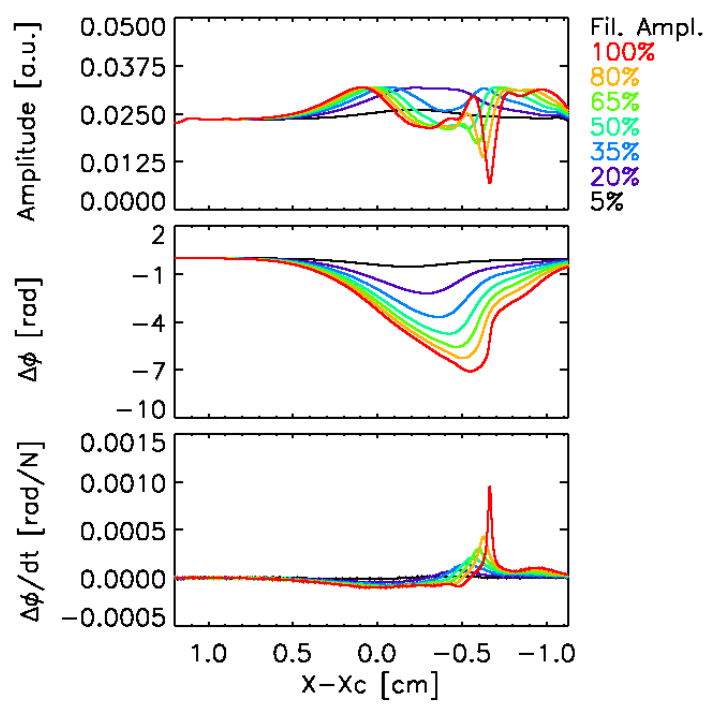

FIG.2. Reflectometry signals for radial propagation of filaments with different density amplitudes $\left(w_{r}=0.3 \mathrm{~cm}\right.$, see fig. 1$)$.

\section{B. Poloidal propagation}

For the poloidal propagation cases we consider $w_{r}=0.9 \mathrm{~cm}$ and a radial location fixed at the cut-off position (approximately the LCFS location in experiments). The filament is a 2D Gaussian perturbation (Gaussian poloidal width $w_{\theta}$ ) and in fig. 3 we 
present the results for a $w_{\theta}$ scan, set as a function of the radial width $w_{r}$.

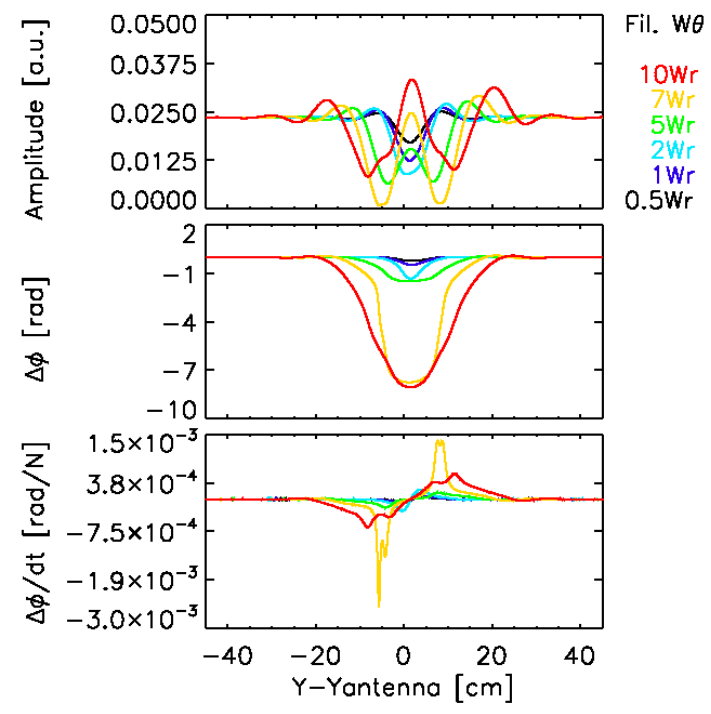

FIG.3. Reflectometry signals for poloidal propagation of filaments with different $w_{\theta}$ ranging in $[0.45-9.0] \mathrm{cm}$. The optical axis of the antenna is taken as reference in the poloidal direction.

In the case of the largest poloidal size $\left(w_{\theta}=9.0 \mathrm{~cm}\right)$ the phase response resembles the Gaussian profile of the perturbation properly describing the cut-off layer displacement and suggesting the 1D geometric optics approximation is valid. At the larger poloidal widths more rapid phase changes occur, as indicated by the phase derivative values. From the whole set of amplitude signals we observe evidence of constructive and destructive interference effects as amplitude dips and peaks emerge depending on the poloidal size of the filaments. A complete analysis of Gaussian perturbations running in poloidal direction has already been made previously ${ }^{10}$.

\section{Diagonal propagation}

For the case of diagonal propagation we consider a filament with Gaussian profiles in both radial and poloidal directions and with equivalent widths $w_{r}=w_{\theta}=0.9 \mathrm{~cm}$. We extend the filament in a certain direction by forcing an additional flat-top length with the filament central density. This creates a larger effective filament poloidal size but is also reasonable experimentally: the toroidal extent and velocity of the filaments combined with the magnetic field line pitch angle can result in a similar effect. The amplitude is set at a constant value of $2 \times 10^{19} \mathrm{~m}^{-3}$. In fig. 4 we show the results obtained with three different angles of propagation and therefore three different ratios of radial to poloidal velocity components. In all cases considered the phase no longer recovers the initial value (a phase jump occurs) and the amplitude signals are intensively modulated while an amplitude dip remains as prominent feature. The phase derivative signal shows how in the case of higher radial velocity component also the phase signal is strongly modulated. Notice that the illumination zone of the reflectometer changes due to the displacement of the cut-off layer and part of the wave can be trapped and then re-emitted which can generate an asymmetry in the reflectometer response.

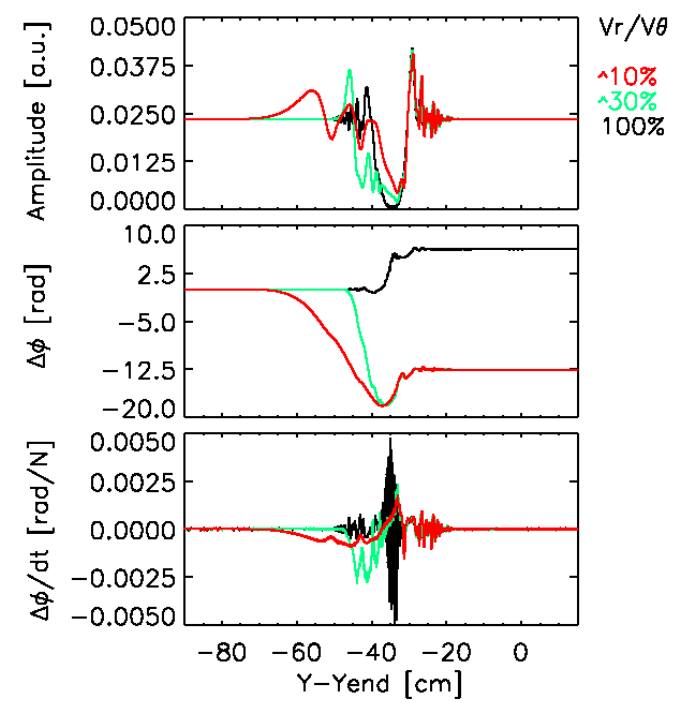

FIG.4. Reflectometry signals for cases of diagonal propagation. The "ending" point of the filaments is shared and taken as reference in the poloidal direction.

\section{DISCUSSION AND CONCLUSIONS}

This study permitted a first insight on the signatures that plasma filaments print on reflectometry signals according to numerical solutions. A method to detect plasma filaments with fixed frequency reflectometry (using a threshold on the time derivative of the phase) has been previously suggested ${ }^{4}$ and the features obtained experimentally and numerically are generally in agreement: filaments outside the LCFS likely travel with both radial and poloidal velocity components thus resulting in phase jumps and amplitude dips. The detection method proposed experimentally should be efficient regardless of the propagation direction, but with a success rate depending on the threshold value and specific filament parameters.

\section{ACKNOWLEDGMENTS}

This work was supported by EURATOM and carried out within the framework of the European Fusion Development Agreement. IST activities also received financial support from Fundação para a Ciência e Tecnologia through project Pest-OE/SADG/LA0010/ 2013. The views and opinions expressed herein do not necessarily reflect those of the European Comission.

${ }^{1}$ A. Kirk et al, Journal of Nuclear Materials 390-391 727 (2009).

${ }^{2}$ H. W. Müller et al, Nucl. Fusion 51073023 (2011).

${ }^{3}$ E. Mazzucato, Rev. Sci. Instrum. 691201 (1998).

${ }^{4}$ J. Vicente, G. D. Conway, H. W. Müller, F. da Silva, S. Da Graça, M. E. Manso and the ASDEX Upgrade team, Proceedings of the 39th EPS Conference on Controlled Fusion and Plasma Physics, Stockholm, 2-6 July 2012, Vol. 36F, P1.031.

${ }^{5}$ F. da Silva, S. Heuraux, S. Hacquin and M. E. Manso, Journal of Computational Physics 203 467-92 (2005).

${ }^{6} \mathrm{R}$. Courant, K. Friedrichs and H. Lewy IBM Journal 11(2) 215-34 (1928)

${ }^{7}$ F. da Silva, S. Heuraux, E. Gusakov and A. Popov, IEEE Trans. Plasma Sci. 38 2144-8 (2010).

${ }^{8}$ L. Cupido et al, Rev. Sci. Instrum. 77320 (2006)

${ }^{9} \mathrm{~F}$. da Silva et al, Nucl. Fusion 46 S816 (2006)

${ }^{10}$ E. Z. Gusakov et al, Plasma Phys. Control. Fusion 441565 (2002) 